\title{
Comparison of Corneal Safety and Intraocular Pressure-Lowering Effect of Tafluprost Ophthalmic Solution with Other Prostaglandin Ophthalmic Solutions
}

\author{
Takeshi Kumagami, ${ }^{1}$ Harumi Wakiyama, ${ }^{2}$ Mao Kusano, ${ }^{1}$ Ayano Kajiyama, ${ }^{1}$ Yoko Miura, \\ Masafumi Uematsu, ${ }^{1}$ Ai Yoneda, ${ }^{2}$ Junko Kurihara, ${ }^{2}$ Kiyoshi Suzuma, and Takashi Kitaoka ${ }^{1}$
}

\begin{abstract}
Purpose: The benzalkonium chloride (BAK) content of tafluprost ophthalmic solution (Tapros ${ }^{\circledR}$ : tafluprost) has been reduced to balance corneal safety and preservative effectiveness (old formulation: $0.01 \%$; new formulation: $0.001 \%$ ). However, no reports have been published on its clinical effect. Therefore, we conducted a clinical research study to compare the safety of BAK-reduced tafluprost on the ocular surface with other prostaglandin ophthalmic solutions.

Methods: This clinical study included 28 glaucoma patients (28 eyes) with a treatment history of latanoprost ophthalmic solution $\left(\right.$ Xalatan ${ }^{\circledR}$ ) or travoprost ophthalmic solution (Travatan $\mathrm{Z}^{\circledR}$ ), who presented with corneal epithelial disorders. The subjects were switched to BAK-reduced tafluprost, and its effect on the ocular surface was examined after 1 and 2 months of treatment [using fluorescein staining score, hyperemia, tear film breakup time, and intraocular pressure (IOP) lowering].

Results: In all analyzed subjects $(N=27)$, the fluorescein staining score was significantly improved after switching to BAK-reduced tafluprost $(P<0.0001)$. Conversely, the IOP-lowering effect was not notably changed. The subjects switched from latanoprost $(n=10)$ showed significant improvement in fluorescein staining score $(P<0.05)$ as well as in IOP lowering $(P<0.01)$. The subjects switched from travoprost $(n=17)$ also showed significant improvement in fluorescein staining score $(P<0.001)$, but without a significant change in IOP lowering.

Conclusions: Tafluprost with reduced BAK has potential as a superior antiglaucoma drug, not only for its IOPlowering effect, but also for its good corneal safety profile.
\end{abstract}

\section{Introduction}

G LAUCOMA OFTEN REQUIRES TOPICAL drug treatment over an extended period because of its chronic nature. If 1 drug is not adequate for treatment, multiple concomitant drugs are often prescribed. Benzalkonium chloride (alkyl dimethylbenzylammonium chloride: BAK), which is included in many ophthalmic drugs as a preservative, is known to be cytotoxic toward corneal epithelial cells, based on the results of in vitro ${ }^{1}$ and in vivo ${ }^{2-4}$ studies. Meanwhile, in the clinic, there are many reports of glaucoma patients developing ocular surface disorders as a result of long-term treatment with multiple glaucoma ophthalmic drugs.

Tafluprost ophthalmic solution $0.0015 \%$ (Tapros ${ }^{\circledR}$; Santen Pharmaceutical Co. Ltd., Osaka, Japan; tafluprost), a prostaglandin analogue for glaucoma treatment, recently had its BAK level optimized ${ }^{5}$ by reduction from the conventional $0.01 \%$ to $0.001 \%$. An in vitro study on cultured human corneal epithelial cells showed that its corneal safety was comparable to a BAK-free tafluprost formulation. ${ }^{6}$ In contrast, $0.005 \%$ latanoprost ophthalmic solution (Xalatan ${ }^{\circledR}$; Pfizer Inc., New York, NY; latanoprost) contains $0.02 \%$ BAK, while $0.004 \%$ travoprost ophthalmic solution (Travatan $Z^{\circledR}$; Alcon Laboratories Inc., Fort Worth, TX; travoprost) contains zinc chloride (sofZia ${ }^{\circledR}$ ) instead of BAK as a preservative. Although previous clinical studies have verified that BAK-free tafluprost shows superior corneal safety compared with latanoprost or travoprost, ${ }^{7-9}$ information on the efficacy and safety of BAK-reduced tafluprost is of clinical importance because it is more widely used in the clinic than preservative-free tafluprost. However, no reports are available to date. The purpose of this study was to assess

\footnotetext{
${ }^{1}$ Department of Ophthalmology and Visual Sciences, Graduate School of Biomedical Sciences, Nagasaki University, Nagasaki, Japan.

${ }^{2}$ Department of Ophthalmology, The Japanese Red Cross Nagasaki Genbaku Hospital, Nagasaki, Japan.
} 
the safety of BAK-reduced tafluprost on the ocular surface and its intraocular pressure (IOP)-lowering effect in glaucoma patients in comparison with other prostaglandin ophthalmic solutions.

\section{Methods}

\section{Patients}

This research study included patients with primary or secondary glaucoma and ocular hypertension, with IOP $\leq$ $30 \mathrm{mmHg}$, who had used latanoprost or travoprost for 3 months or longer and presented with superficial punctate keratitis (SPK) identified by fluorescein corneal staining. Patients were excluded based on the following criteria: $<20$ years of age; use of more than 2 concomitant nonprostaglandin ophthalmic drugs for treatment of diseases other than glaucoma; presence of active extraocular disease, including allergies, inflammation of the eye or eyelids, infections, or diabetes, which may interfere with assessment of improvement in corneal epithelial disorders; requirement for contact lens wear; history of corneal refractive surgery or corneal transplant; or history of intraocular or extraocular surgery within 3 months of the study.

The patients provided informed consent following a full explanation using appropriate documents. The study was conducted in accordance with the Declaration of Helsinki, and approved by the Nagasaki University Hospital Clinical Research Investigational Review Board, as well as the Nagasaki Genbaku Hospital Clinical Research Committee.

\section{Methods}

Superficial punctate keratitis, an indicator of corneal epithelial injury, was selected as the primary endpoint, and the NEI/I Method ${ }^{10}$ was used for assessment. In the NEI/I Method, the cornea is divided into five sections: central; superior; temporal; nasal; and inferior. The density of fluorescein punctate staining in each section was scored as follows: $0=$ none; $1=$ sparse; $2=$ dense; and $3=$ coalesced. For conjunctival hyperemia, a 5-point grading scale $(0=$ none; $1=$ mild; $2=$ moderate; $3=$ severe; and $4=$ very severe) was used. $^{11,12}$ The cornea or tear fluid was stained with fluorescein test paper, and observations were made of SPK and tear film breakup time (BUT) under a slitlamp microscope. For all evaluations, the same investigator examined the same subject throughout the study period. Corneal staining score and conjunctival hyperemia were assessed using grading scales, and single grading tables with reference photographs were used to unify the evaluation criteria. If both eyes were eligible, the eye with the higher fluorescein staining score was selected as the study eye (if the scores were the same, the right eye was selected). If the subject used only 1 concomitant nonprostaglandin glaucoma ophthalmic drug, it was continued. If the subject had been receiving drug treatment for a corneal epithelial disorder, such as sodium hyaluronate ophthalmic solution (HA), it was discontinued at the beginning of the study. IOP was measured with an applanation tonometer, with the measurements taken in an unblinded manner.

\section{Statistical analysis}

For statistical analyses, the data before and after switching to BAK-reduced tafluprost were compared. The fluo- rescein staining score and IOP were analyzed by a paired $t$-test, and BUT and conjunctival hyperemia were analyzed by the Wilcoxon signed-rank test. The significance level was $5 \%$ on both sides. JMP 10.0 (SAS Institute, Tokyo, Japan) was used as the analytical software.

\section{Results}

\section{Patient distribution and background}

Of the 28 subjects (28 eyes) enrolled, 7 subjects were taking HA for a corneal epithelial disorder and 2 subjects (2 eyes) discontinued the study. Both of these subjects had been receiving treatment for a corneal epithelial disorder (Hyalein $0.1 \%{ }^{\circledR}$; Santen Pharmaceutical Co. Ltd., Osaka, Japan). One discontinued the study because worsening of the SPK score at week 4, and the data for this subject were included in the statistical analyses. The other subject was not included in the statistical analyses because the patient withdrew from the study, and there were no data available after tafluprost treatment. Consequently, 27 subjects (27 eyes) were included in the final analysis and their details are shown in Table 1 . The study group consisted of 12 males and 15 females, with a mean age $( \pm S D)$ of $65.3 \pm 12.0$ years. The glaucoma types were as follows: primary openangle glaucoma, 8 subjects; normal tension glaucoma, 16 subjects; angle closure glaucoma, 2 subjects; and exfoliation glaucoma, 1 subject. Ten subjects (10 eyes) were switched from latanoprost and 17 subjects (17 eyes) were switched from travoprost. In the final analysis set, 6 subjects (6 eyes) were treated for a corneal epithelial disorder. Six subjects

Table 1. Patient Demographics $(N=27)$

\begin{tabular}{lc}
\hline Sex & \\
Male & $12(44 \%)$ \\
Female & $15(56 \%)$ \\
Age & \\
Mean (y) & $65.3 \pm 12.0$ \\
MD & \\
Mean, dB & \\
Corneal thickness & $5.18 \pm 5.50$ \\
$\quad$ Mean, $\mu \mathrm{m}$ & $531.39 \pm 35.6$ \\
Ocular diagnosis & $8(31 \%)$ \\
POAG & $16(59 \%)$ \\
NTG & $2(7 \%)$ \\
PACG & $1(4 \%)$ \\
PEX & $10(37 \%)$ \\
Prior prostaglandin & $17(63 \%)$ \\
Latanoprost (Xalatan 0.005\%) & \\
Travoprost (Travatan Z 0.004\%) & $21(78 \%)$ \\
Concomitant medicine with prostaglandin & $3(11 \%)$ \\
No use & $2(7 \%)$ \\
Carteolol (Mikelan LA 1\%) & $1(4 \%)$ \\
Bunazosin (Detantol 0.01\%) & \\
Timolol (Rysmon TG $0.5 \%)$ & $6(78 \%)$ \\
Prior medication for corneal epithelium disorder & \\
No use & $6(22 \%)$ \\
Sodium hyaluronate (Hyalein 0.1\%) & \\
\hline
\end{tabular}

Values are shown as the number $(\%)$ of patients or mean \pm SD.

${ }^{\mathrm{a}}$ For measurements of visual fields by automated perimetry, Humphrey program 24-2 was used.

MD, mean defect; POAG, primary open-angle glaucoma; NTG, normal tension glaucoma; PACG, primary angle closure glaucoma; PEX, primary exfoliation glaucoma. 
used concomitant antiglaucoma drugs, with 3 subjects taking $1 \%$ carteolol ophthalmic solution (Mikelan LA ${ }^{\circledR}$; Otsuka Pharmaceutical Co. Ltd., Tokyo, Japan), 2 subjects taking bunazosin ophthalmic solution (Detantol ${ }^{\circledR}$; Santen Pharmaceutical Co. Ltd., Osaka, Japan), and 1 subject taking $0.5 \%$ timolol ophthalmic solution (Rysmon $\mathrm{TG}^{\circledR}$; Kissei Pharmaceutical Co. Ltd., Matsumoto, Japan).

\section{Safety and efficacy}

Fluorescein staining scores. In all 27 analyzed subjects, the staining scores (mean $\pm \mathrm{SE}$ ) at the beginning of treatment (baseline), and after 1 and 2 months of treatment were $3.67 \pm 0.40,2.33 \pm 0.40$, and $1.88 \pm 0.30$, respectively. After 1 and 2 months of treatment, significant decreases in the staining scores $(P<0.0001)$ were found compared with baseline (Fig. 1A). In the subjects switched from latanoprost $(n=10)$, the staining scores at baseline, and after 1 and 2 months of treatment were $3.90 \pm 0.70,2.80 \pm 0.80$, and $2.56 \pm 0.70$, respectively, with the staining score after 2 months of treatment showing a significant decrease $(P<0.05)$ compared with baseline (Fig. 1B). Furthermore, in

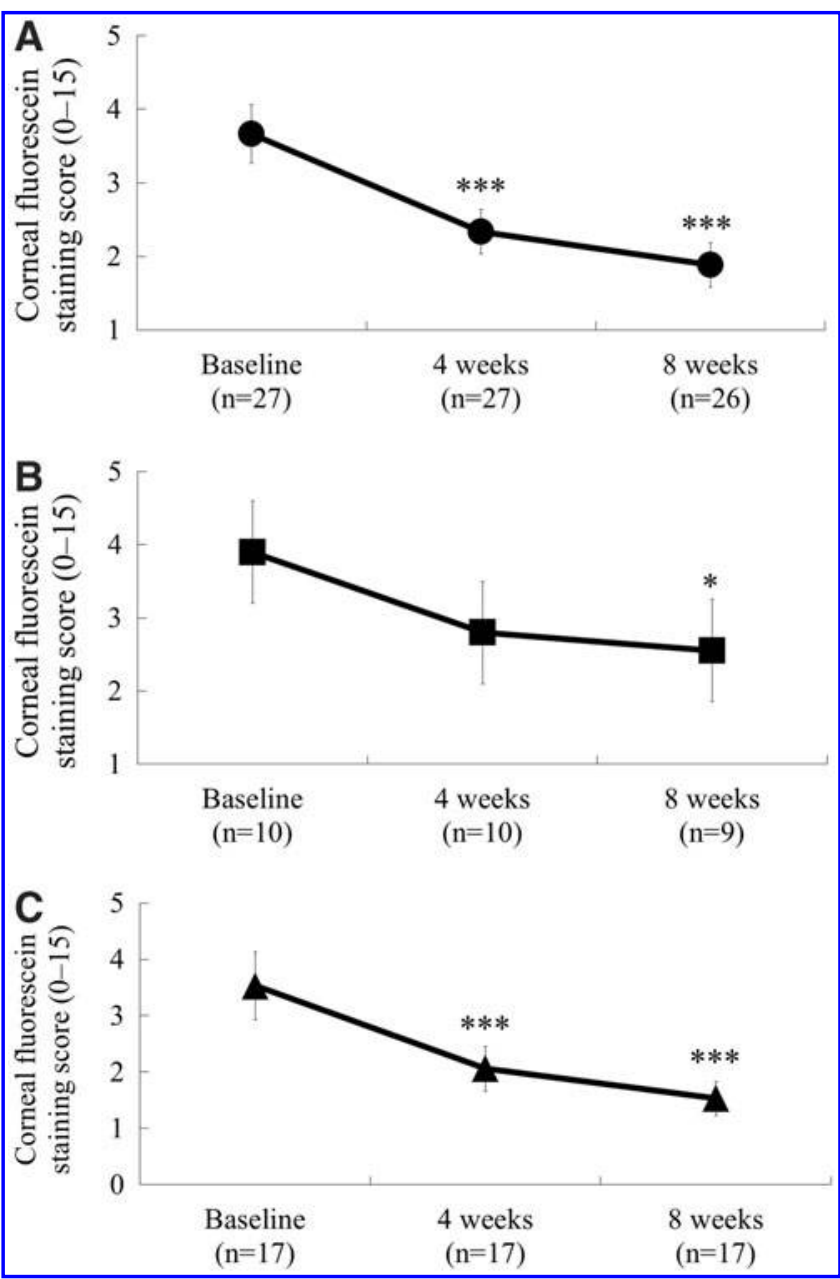

FIG. 1. Time-course changes in the fluorescein staining scores. (A) Time-course changes in all subjects. (B) Timecourse changes in subjects switched from latanoprost. (C) Time-course changes in subjects switched from travoprost. Data are shown as means $\pm \mathrm{SE}(* P<0.05, * * * P<0.001$ vs. baseline, paired $t$-test). the subjects switched from travoprost $(n=17)$, the staining scores at baseline, and after 1 and 2 months of treatment were $3.53 \pm 0.60,2.06 \pm 0.40$, and $1.53 \pm 0.30$, respectively, with the staining scores after 1 and 2 months of treatment showing significant decreases $(P<0.0001)$ compared with baseline (Fig. 1C). Corneal staining scores improved in $78 \%$ $(21 / 27)$, were unchanged in $15 \%(4 / 27)$, and worsened in $7 \%(2 / 27)$ (Fig. 2A). In the group switched from latanoprost $(n=10), 70 \%(7 / 10)$ had improved, 10\% (1/10) were unchanged, and $20 \%(2 / 10)$ had worsened (Fig. 2B). In the group switched from travoprost $(n=17), 76 \%(13 / 17)$ had improved, 24\% (4/17) were unchanged, and $0 \%(0 / 17)$ had worsened (Fig. 2C).

Conjunctival hyperemia scores. In all the analyzed subjects, the conjunctival hyperemia scores (mean $\pm \mathrm{SE}$ ) at baseline, and after 1 and 2 months of treatment were $0.52 \pm 0.10,0.44 \pm 0.10$, and $0.35 \pm 0.1$, respectively, showing a decreasing trend compared with baseline, although the difference was not statistically significant $(P=0.063)$. In the subjects switched from latanoprost, the conjunctival hyperemia scores at baseline, and after 1 and 2 months of treatment were $0.30 \pm 0.20,0.40 \pm 0.20$, and $0.33 \pm 0.20$, respectively, with no significant differences compared with baseline. In the subjects switched from travoprost, the conjunctival hyperemia scores at baseline, and after 1 and 2 months of treatment were $0.65 \pm 0.20,0.47 \pm 0.20$, and $0.35 \pm 0.10$, respectively, with the score after 2 months of treatment showing a decreasing trend compared with baseline, although the difference was not statistically significant $(P=0.063)$.

BUT. In all the analyzed subjects, the BUT values (mean $\pm \mathrm{SE}$ ) at baseline, and after 1 and 2 months of treatment were $5.41 \pm 0.40,5.67 \pm 0.50$, and $5.69 \pm 0.50$ seconds, respectively, with no significant differences compared with baseline. In the subjects switched from latanoprost, the BUT values at baseline, and after 1 and 2 months of treatment

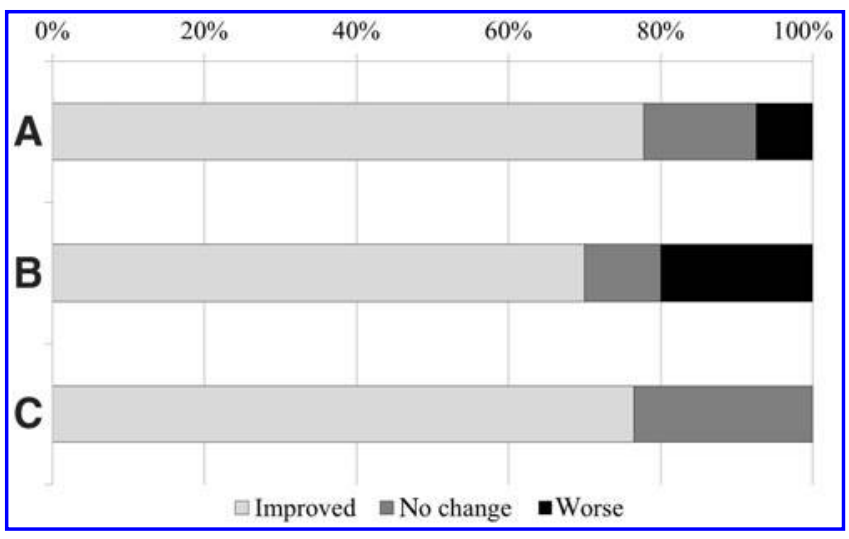

FIG. 2. Changes in the fluorescein staining scores after 8 weeks of tafluprost treatment. Improved: fluorescein staining score improved by 1 or more compared with baseline. No change: fluorescein staining score is the same as baseline. Worse: fluorescein staining score has worsened by 1 or more compared with baseline. (A) Time-course changes in all subjects. (B) Time-course changes in subjects switched from latanoprost. (C) Time-course changes in subjects switched from travoprost. 
were $5.20 \pm 0.90,5.20 \pm 0.80$, and $5.33 \pm 0.90$, respectively, with no significant differences compared with baseline. In the subjects switched from travoprost, the BUT values at baseline, and after 1 and 2 months of treatment were $5.53 \pm 0.50,5.94 \pm 0.60$, and $5.88 \pm 0.60$, respectively, with no significant differences compared with baseline.

Treatment drugs for corneal epithelial disorders. There were 7 subjects who had been using a concomitant treatment drug for a corneal epithelial disorder (hyaluronic acid ophthalmic solution). In these subjects, the corneal staining scores improved in 57\% (4/7 subjects), remained unchanged in $14 \%$ ( $1 / 7$ subjects), and worsened in $29 \%$ ( $2 / 7$ subjects). Therefore, in 5 of 7 (71\%) subjects under HA treatment for a corneal epithelial disorder at baseline, no worsening of the corneal epithelial disorder was seen after switching to tafluprost. Consequently, we were able to discontinue treatment for the corneal epithelial disorder in these subjects.

IOP. In all the analyzed subjects, the IOP values (mean \pm SE) at baseline, and after 1 and 2 months of treatment were $12.74 \pm 0.40,12.33 \pm 0.40$, and $12.19 \pm$ $0.40 \mathrm{mmHg}$, respectively, with no significant changes compared with baseline (Fig. 3A). In the subjects switched from latanoprost, the IOP values at baseline, and after 1 and 2 months of treatment were $12.80 \pm 0.80,12.80 \pm 0.90$, and $11.44 \pm 0.70 \mathrm{mmHg}$, respectively, with the value after 2 months of treatment showing a significant IOP-lowering effect $(P>0.01)$ compared with baseline (Fig. 3B). In the subjects switched from travoprost, the IOP values at baseline, and after 1 and 2 months of treatment were $12.71 \pm 0.50,12.06 \pm 0.50$, and $12.59 \pm 0.50 \mathrm{mmHg}$, respectively, with no significant changes compared with baseline (Fig. 3C).

\section{Discussion}

In glaucoma drug preparations, it is possible for the active drug itself to affect the ocular surface, rather than just the preservatives. However, in a previous study, human primary subcutaneous pre-adipocytes were exposed to 4 prostaglandin products (bimatoprost, travoprost, latanoprost, and tafluprost), and the formulations themselves did not display any cytotoxicity. ${ }^{13}$ In addition, preservative-free prostaglandin ophthalmic solutions have been shown to exhibit very low cytotoxicity against corneal epithelial cells. ${ }^{14}$ These findings suggest that the excipients, primarily preservatives, play a major role in the corneal epithelial disorders caused by prostaglandin ophthalmic solutions.

The results of the present study indicate that corneal epithelial disorders were alleviated with BAK-reduced tafluprost in comparison with latanoprost or travoprost. The subjects who were switched from latanoprost to BAKreduced tafluprost showed significant improvement in their corneal disorder, largely because the BAK concentration was one-twentieth of its level in latanoprost. This is similar to the results of previous studies by Uusitalo et al. ${ }^{7}$ and Janulevičiene et al., ${ }^{8}$ who compared the effects of latanoprost and BAK-free tafluprost. In particular, it should be noted that the corneal disorder was alleviated in subjects who were switched from travoprost to BAK-reduced tafluprost. There have been reports that sofZia ${ }^{\circledR}$-containing travoprost has a superior safety profile for the ocular surface

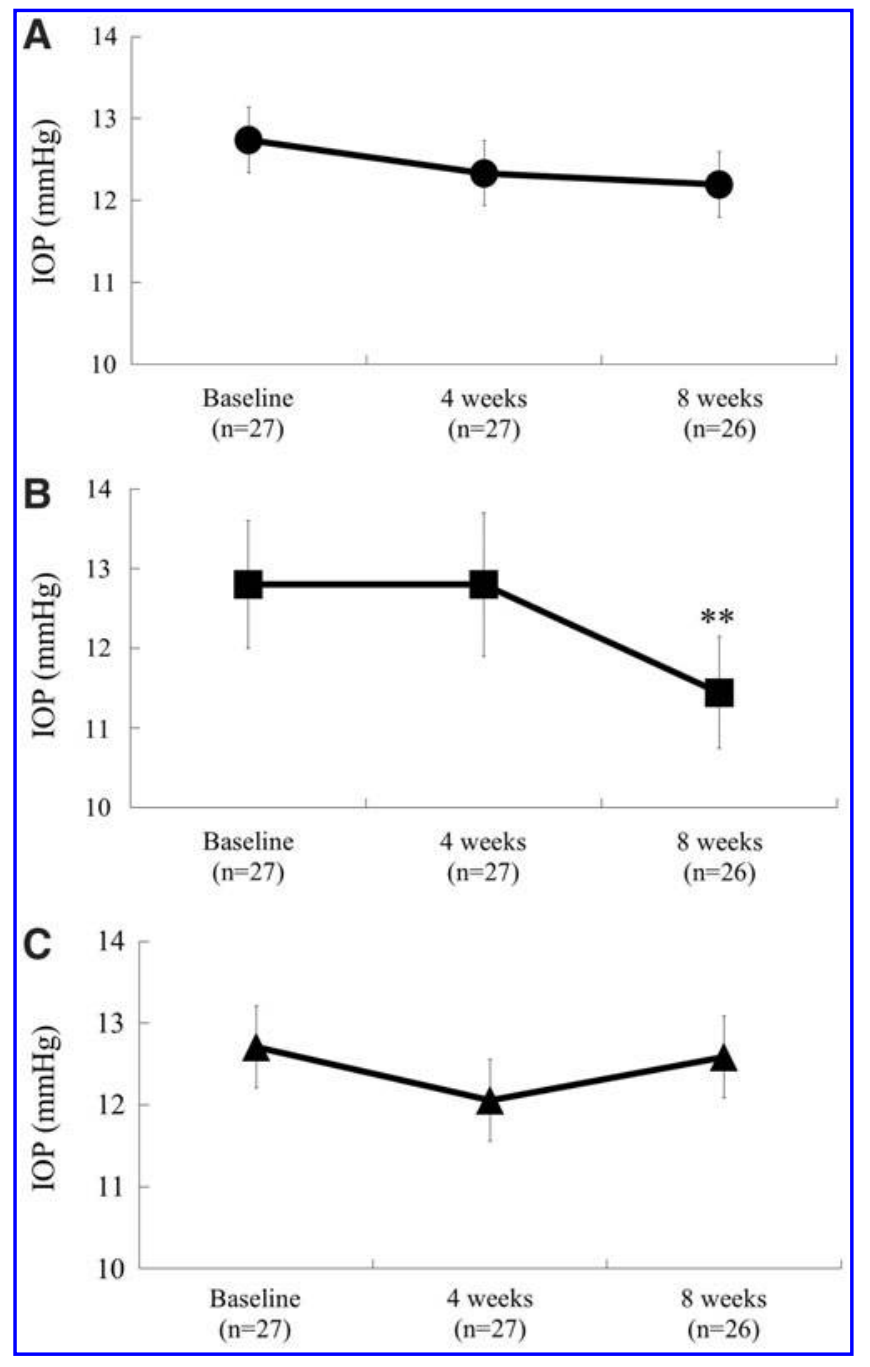

FIG. 3. Time-course changes in the intraocular pressure (IOP). (A) IOP changes in all subjects. (B) Time-course changes in subjects switched from latanoprost. (C) Timecourse changes in subjects switched from travoprost. Data are shown as means $\pm \mathrm{SE}(* * P<0.01$ vs. baseline, paired $t$-test).

compared with BAK-containing latanoprost. ${ }^{15-18}$ However, the present study showed that BAK-reduced tafluprost caused less corneal injury than travoprost, thereby indicating that the adverse effects of BAK on the cornea can be alleviated by lowering its concentration, while maintaining its effectiveness as a preservative. In the present study, tafluprost showed better corneal safety than travoprost, which could be attributed to their different cytotoxicities against corneal epithelial cells. In a study using cultured corneal epithelial cells, tafluprost showed minimal cytotoxicity, equivalent to preservative-free tafluprost. ${ }^{6}$ On the other hand, another report described that travoprost was slightly cytotoxic. ${ }^{14}$ The results from these in vitro studies indicate that tafluprost has lower cytotoxicity than travoprost. Based on the results of the present study, we believe that switching from travoprost to tafluprost alleviated the toxicity against corneal epithelial cells, resulting in the decreased SPK scores.

Regarding IOP lowering (the most common effect of antiglaucoma drugs), tafluprost with the reduced preservative content caused significant IOP lowering in subjects switched from latanoprost, while no changes were seen in 
subjects switched from travoprost. Ranno et al. ${ }^{19}$ reported that switching from latanoprost or travoprost to BAK-free tafluprost did not cause any IOP-lowering effects. Conversely, Hommer et al. ${ }^{9}$ reported that, in patients with insufficient IOP lowering and subjects who had developed adverse drug reactions (ADRs) in the ocular surface, a further IOP-lowering effect was found by switching from latanoprost to BAK-free tafluprost, and these findings were similar to the results of the present study. The discrepancy may be attributed to the difference in the subject selection methods. As found in the study by Hommer et al. ${ }^{9}$ and the present study, there is a possibility that, in patients who have ocular surface ADRs and who are switched to a drug that alleviates the ADR symptoms, there is improved drug adherence, which would result in further IOP-lowering effects. However, in the present study, the subjects switched from travoprost did not show improved IOP lowering. This may be clarified by further investigations. Nevertheless, in the present study, the IOP-lowering effect of BAK-reduced tafluprost was noninferior to latanoprost or travoprost. In the present study, among the subjects who were under treatment for a corneal epithelial disorder at baseline, $71 \%$ (5/7) were able to discontinue concomitant corneal disorder treatment drugs (HA). BAK-reduced tafluprost may also enable glaucoma patients to reduce the total number of concomitant ophthalmic solutions. Although increased instillation frequency and negative adherence are not always correlated, ${ }^{20,21}$ decreasing the number of ophthalmic drugs should contribute to relieving the burden on patients. ${ }^{22}$

In $75 \%(21 / 28)$ of the enrolled subjects, the SPK scores improved by switching their glaucoma drug to tafluprost. Conversely, the SPK scores worsened in $11 \%(3 / 28)$. Of the 3 subjects with worsening of the SPK scores, 2 subjects were using HA for treatment of their corneal epithelial disorder at baseline, and these 2 subjects discontinued HA upon starting tafluprost treatment. Therefore, we believe there is a high possibility that the scores in these patients worsened through discontinuation of the treatment for their corneal epithelial disorder. On the other hand, 1 subject with worsening of the SPK score was switched from latanoprost, and the reason for the worsening remains unclear. The above results indicate that, in a small portion of subjects, SPK would be worsened by switching to tafluprost.

Since the present study aimed to investigate the effect of tafluprost on corneal epithelial disorders, there was no assessment of subjective symptoms. Therefore, it remains unknown whether such symptoms would be improved by switching the treatment drug to tafluprost. Subjective symptom improvement is a very important factor that should be investigated in future studies, because an improvement would lead to improved quality of life for patients, as well as better treatment compliance. We are hoping that the corneal epithelial disorder improvement achieved by switching the treatment drug to tafluprost would also lead to improvement of subjective symptoms. In addition, since the results of the present study were based on patients who presented with SPK, future studies should investigate the effect on patients without SPK. Finally, this study was an exploratory study assessing a very small patient population with a short-term evaluation period ( 2 months), and the results need to be verified in a large-scale, long-term study.

In conclusion, tafluprost with a reduced preservative content, compared with latanoprost or travoprost, may have an improved corneal safety profile, while maintaining a potent IOP-lowering effect.

\section{Author Disclosure Statement}

No competing financial interests exist.

\section{References}

1. Neville, R., Dennis, P., Sens, D., and Crouch, R. Preservative cytotoxicity to cultured corneal epithelial cells. Curr. Eye Res. 5:367-372, 1986.

2. Uematsu, M., Kumagami, T., Shimoda, K., et al. Influence of alkyl chain length of benzalkonium chloride on acute corneal epithelial toxicity. Cornea 29:1296-1301, 2010.

3. Uematsu, M., Kumagami, T., and Kusano, M., et al. Acute corneal epithelial change after instillation of benzalkonium chloride evaluated using a newly developed in vivo corneal transepithelial electric resistance measurement method. Ophthalmic Res. 39:308-314, 2007.

4. Araki, R., Nakashima, M., and Teshima, M., et al. Investigation of protective effects of sodium hyaluronate eyedrop against corneal epithelial disorders using an electrophysiological method. J. Ocul. Pharmacol. Ther. 28: 251-258, 2012.

5. Asada, H., Takaoka-Shichijo, Y., Nakamura, M., and Kimura, A. Optimization of benzalkonium chloride concentration in $0.0015 \%$ tafluprost ophthalmic solution from the points of ocular surface safety and preservative efficacy. Yakugaku Zasshi 130:867-871, 2010 (in Japanese).

6. Nakagawa, S., Usui, T., and Yokoo, S., et al. Toxicity evaluation of antiglaucoma drugs using stratified human cultivated corneal epithelial sheets. Invest. Ophthalmol. Vis. Sci. 53:5154-5160, 2012.

7. Uusitalo, H., Chen, E., Pfeiffer, N., et al. Switching from a preserved to a preservative-free prostaglandin preparation in topical glaucoma medication. Acta. Ophthalmol. 88:329$336,2010$.

8. Janulevičienè, I., Derkač, I., Grybauskiene, L., et al. Effects of preservative-free tafluprost on tear film osmolarity, tolerability, and intraocular pressure in previously treated patients with open-angle glaucoma. Clin. Ophthalmol. 6: 103-109, 2012.

9. Hommer, A., and Kimmich, F. Switching patients from preserved prostaglandin-analog monotherapy to preservative-free tafluprost. Clin. Ophthalmol. 5:623-631, 2011.

10. Lemp, M.A. Report of the National Eye Institute/Industry workshop on Clinical Trials in Dry Eyes. CLAO J. 21:221232, 1995.

11. Papas, E.B. Key factors in the subjective and objective assessment of conjunctival erythema. Invest. Ophthalmol. Vis. Sci. 41:687-691, 2000.

12. Murphy, P.J., Lau, J.S., Sim, M.M., and Woods, R.L. How red is a white eye? Clinical grading of normal conjunctival hyperemia. Eye (Lond) 21:633-638, 2007.

13. Seibold, L.K., Ammar, D.A., and Kahook, M.Y. Acute effects of glaucoma medications and benzalkonium chloride on pre-adipocyte proliferation and adipocyte cytotoxicity in vitro. Curr. Eye Res. 38:70-74, 2013.

14. Pellinen, P., Huhtala, A., Tolonen, A., et al. The cytotoxic effects of preserved and preservative-free prostaglandin analogs on human corneal and conjunctival epithelium in vitro and the distribution of benzalkonium chloride homologs in ocular surface tissues in vivo. Curr. Eye Res. 37:145-154, 2012. 
15. Kahook, M.Y., and Noecker, R.J. Comparison of corneal and conjunctival changes after dosing of travoprost preserved with sofZia, latanoprost with $0.02 \%$ benzalkonium chloride, and preservative-free artificial tears. Cornea 27: 339-343, 2008.

16. Henry, J.C., Peace, J.H., Stewart, J.A., and Stewart, W.C. Efficacy, safety, and improved tolerability of travoprost BAK-free ophthalmic solution compared with prior prostaglandin therapy. Clin. Ophthalmol. 2:613-621, 2008.

17. Yamazaki, S., Nanno, M., Kimura, T., Suzumura, H., and Yoshikawa, K. Effects of switching to SofZia-preserved travoprost in patients who presented with superficial punctate keratopathy while under treatment with latanoprost. Jpn. J. Ophthalmol. 54:7-14, 2010.

18. Aihara, M., Oshima, H., Araie, M., EXTraKT study group. Effects of SofZia-preserved travoprost and benzalkonium chloride-preserved latanoprost on the ocular surface-a multicentre randomized single-masked study. Acta. Ophthalmol. 91:e7-e14, 2013.

19. Ranno, S., Sacchi, M., Brancato, C., et al. A prospective study evaluating IOP changes after switching from a therapy with prostaglandin eye drops containing preservatives to nonpreserved tafluprost in glaucoma patients. Sci. World J. 2012:[Epub ahead of print]; DOI: 10.1100/2012/804730.
20. Robin, A.L., Novack, G.D., Covert, D.W., Crockett, R.S., and Marcic, T.S. Adherence in glaucoma: objective measurements of once-daily and adjunctive medication use. Am. J. Ophthalmol. 144:533-540, 2007.

21. Djafari, F., Lesk, M.R., Harasymowycz, P.J., Desjardins, D., and Lachaine, J. Determinants of adherence to glaucoma medical therapy in a long-term patient population. J. Glaucoma 18:238-243, 2009.

22. Takahashi, M., Naitou, T., Mizoue, S., et al. Questionnaire survey on use of glaucoma eyedrops: second report. Atarashii Ganka 29:555-561, 2012 (in Japanese).

Received: July 22, 2013

Accepted: December 22, 2013

Address correspondence to:

Dr. Takeshi Kumagami

Department of Ophthalmology and Visual Sciences Graduate School of Biomedical Sciences Nagasaki University 1-7-1 Sakamoto Nagasaki 852-8501

Japan

E-mail: kumagami-t@umin.ac.jp 